\title{
On generalized Fibonacci quadratics
}

\author{
Neşe Ömür ${ }^{1}$ and Zehra Betül Gür ${ }^{2}$ \\ ${ }^{1}$ Department of Mathematics, University of Kocaeli \\ 41380 İzmit Kocaeli, Turkey \\ e-mail: neseomur@gmail.com \\ 2 Department of Mathematics, University of Kocaeli \\ 41380 İzmit Kocaeli, Turkey \\ e-mail: gurbetul@gmail.com
}

Received: 2 January 2020

Revised: 6 April 2020

Accepted: 22 April 2020

Abstract: In this paper, we consider generalized Fibonacci quadratics and give solutions of them under certain conditions. For example, for odd number $k$, under condition $n=U_{k}^{2}\left(V_{k} V_{k(4 n+1)}-4\right)$, the equation

$$
n x^{2}+\left(V_{k} n-2 U_{k}^{2} D\right) x-\left(n+D U_{k}^{2}\left(V_{k}+2\right)\right)=0
$$

has rational roots.

Keywords: Generalized Fibonacci numbers, Fibonacci quadratics, Pythagorean triplet.

2010 Mathematics Subject Classification: 11B39, 11B50, 97F40.

\section{Introduction}

The second order sequence $\left\{W_{n}(a, b ; p, q)\right\}$, or briefly $\left\{W_{n}\right\}$ is defined for $n>2$ by

$$
W_{n}=p W_{n-1}+q W_{n-2},
$$

in which $W_{0}=a, W_{1}=b$, where $a, b$ are arbitrary integers and $p, q$ are nonzero integers [1]. The Binet formula for $\left\{W_{n}\right\}$ is

$$
W_{n}=A \alpha^{n}+B \beta^{n},
$$

where $A=\frac{b-a \beta}{\alpha-\beta}, B=\frac{a \alpha-b}{\alpha-\beta}$ and $\alpha, \beta=\left(p \pm \sqrt{p^{2}+4 q}\right) / 2$. 
In $[3,4]$, E. Kılıç and P. Stanica derived the following recurrence relation for the sequence $\left\{W_{k n}\right\}$. For $n>2$ and a fixed positive integer $k$,

$$
W_{k n}=V_{k} W_{k(n-1)}-(-q)^{k} W_{k(n-2)},
$$

where $V_{k}=\alpha^{k}+\beta^{k}$. Specifically define the generalized Fibonacci $\left\{U_{n}\right\}$ and Lucas $\left\{V_{n}\right\}$ sequences as $U_{n}=W_{n}(0,1 ; p, 1), V_{n}=W_{n}(2, p ; p, 1)$, respectively. Thus;

$$
\begin{aligned}
U_{k n} & =V_{k} U_{k(n-1)}+(-1)^{k+1} U_{k(n-2)}, \\
V_{k n} & =V_{k} V_{k(n-1)}+(-1)^{k+1} V_{k(n-2)} .
\end{aligned}
$$

The Binet formulas are

$$
U_{k n}=\frac{\alpha^{k n}-\beta^{k n}}{\alpha-\beta} \text { and } V_{k n}=\alpha^{k n}+\beta^{k n},
$$

respectively. The equations

$$
\begin{aligned}
& a x^{2}+b x-c=0, a x^{2}-b x-c=0, \\
& c x^{2}+b x-a=0, \quad c x^{2}-b x-a=0
\end{aligned}
$$

have the same discriminant and then, the first one is considered. Rational roots of the quadratic equation $a x^{2}+b x+c=0$ are given under certain conditions.

In [7], the author gave that the solutions of the equation $F_{n} x^{2}+F_{n+1} x-F_{n+2}=0$ are -1 and $F_{n+2} / F_{n}$.

In [6], for $m \in \mathbb{Z}^{+}$, the author gave the rational solutions of the three equations under conditions $n=F_{2 m+1}-1, F_{2 m+3} F_{2 m}$ and $F_{2 m+1} F_{2 m}$, respectively:

$$
\begin{aligned}
& n x^{2}+(n+1) x-(n+2)=0, \\
& n x^{2}+(n+2) x-(n+1)=0, \\
& n x^{2}+(n-1) x-(n+1)=0 .
\end{aligned}
$$

In [5], for $n, r \in \mathbb{Z}^{+}$, the authors obtained the solutions of equations

$$
\begin{aligned}
n x^{2}+(n+r) x-(n+2 r) & =0, \\
n x^{2}+(n+2 r) x-(n+r) & =0, \\
n x^{2}+(n-r) x-(n+r) & =0 .
\end{aligned}
$$

In this paper, we consider generalized Fibonacci quadratics and give solutions of them under certain conditions. For example, for odd number $k$, under condition $n=U_{k}^{2}\left(V_{k} V_{k(4 n+1)}-4\right)$, the equation

$$
n x^{2}+\left(V_{k} n-2 U_{k}^{2} D\right) x-\left(n+D U_{k}^{2}\left(V_{k}+2\right)\right)=0
$$

has rational roots.

\section{Generalized Fibonacci quadratics}

Throughout this paper, we denote $D=V_{k}^{2}+4$. We will consider some interesting quadratics including generalized Fibonacci numbers and give the solutions of them under conditions $\frac{U_{k}^{2}}{D}\left(4-V_{k} V_{k(4 n-1)}\right), \frac{U_{k}^{2}}{D}\left(V_{k} V_{k(4 n+3)}-4\right)$ and $U_{k}^{2}\left(V_{k} V_{k(4 n+1)}-4\right)$, respectively. 
Lemma 2.1. For odd number $k$, we have

$$
\begin{aligned}
U_{k n} V_{k m}+V_{k n} U_{k m} & =2 U_{k(n+m)}, \\
U_{k n} V_{k m}-V_{k n} U_{k m} & =-2 U_{k(n-m)}, \\
U_{k}^{2} V_{k n} V_{k m}+D U_{k n} U_{k m} & =2 U_{k}^{2} V_{k(n+m)}, \\
V_{k(n+m)}+V_{k(n-m)} & =\left\{\begin{array}{cc}
\frac{D}{U_{k}^{2}} U_{k n} U_{k m} & \text { if } m \text { is odd } \\
V_{k n} V_{k m} & \text { if } m \text { is even }
\end{array} .\right.
\end{aligned}
$$

Proof. From the Binet formulas of sequences $\left\{U_{k n}\right\}$ and $\left\{V_{k n}\right\}$, we have the desired identities.

In [2], the authors determined Pell equations involving the generalized Fibonacci and Lucas sequences by the following Lemmas:

Lemma 2.2. For odd number $k$, the integer solutions of $D x^{2}+4 U_{k}^{2}=y^{2} U_{k}^{2}$ are precisely the pairs $\left( \pm U_{2 k n}, \pm V_{2 k n}\right)$.

Lemma 2.3. For odd number $k$, the integer solutions of $D x^{2}-4 U_{k}^{2}=y^{2} U_{k}^{2}$ are precisely the pairs $\left( \pm U_{k(2 n+1)}, \pm V_{k(2 n+1)}\right)$.

Lemma 2.4. For odd number $k$, the integer solutions of $D U_{k}^{2}\left(x^{2}-4\right)=y^{2}$ are precisely the pairs $\left( \pm V_{2 k n}, \pm D U_{2 k n}\right)$.

Theorem 2.5. For odd number $k$, rational solutions to

$$
n x^{2}+\left(n V_{k}+2 U_{k}^{2}\right) x-\left(n-U_{k}^{2}\left(V_{k}+2\right)\right)=0
$$

exist if and only if $n=\frac{U_{k}^{2}}{D}\left(4-V_{k} V_{k(4 n-1)}\right)$ and they are

$$
-\frac{V_{k}}{2}-\frac{D}{4-V_{k} V_{k(4 n-1)}} \pm \frac{D V_{k} U_{k(4 n-1)}}{2 U_{k}\left(4-V_{k} V_{k(4 n-1)}\right)} .
$$

Proof. The discriminant of (2) is

$$
\Delta=V_{k}^{2} n^{2}+4\left(U_{k}^{2}-n\right)^{2} .
$$

Rational solutions of (2) exist if and only if $\Delta$ is a perfect square. Hence,

$$
\begin{aligned}
& n^{2} V_{k}^{2}+4\left(U_{k}^{2}-n\right)^{2}=V_{k}^{2} t^{2} \\
& n^{2}+4\left(\frac{U_{k}^{2}}{V_{k}}-\frac{n}{V_{k}}\right)^{2}=t^{2} .
\end{aligned}
$$

Thus, the Pythagorean triplet has $\left[n, \frac{2\left(-n+U_{k}^{2}\right)}{V_{k}}, t\right]$, not necessarily primitive. If we present the triplet as

$$
\left[g^{2}-h^{2}, 2 g h, g^{2}+h^{2}\right]
$$

then

and hence

$$
n=g^{2}-h^{2}, \quad \frac{U_{k}^{2}}{V_{k}}-\frac{n}{V_{k}}=g h, \quad t=g^{2}+h^{2},
$$

$$
g^{2}+V_{k} h g-h^{2}-U_{k}^{2}=0
$$


From the discriminant of this equation, we have

$$
D h^{2}+4 U_{k}^{2}=U_{k}^{2} s^{2}
$$

Then this equation has positive solutions $h=U_{2 k n}$ and $s=V_{2 k n}$ with $n \geq 1$ in Lemma 2.2. Hence

$$
g=\frac{-h V_{k} \pm U_{k} s}{2}=\frac{-U_{2 k n} V_{k} \pm U_{k} V_{2 k n}}{2}
$$

and taking $m=1$ and $2 n$ instead of $n$ in Lemma 2.1,

$$
\begin{aligned}
& g=\frac{-U_{2 k n} V_{k}+U_{k} V_{2 k n}}{2}=U_{k(2 n-1)}, \\
& g=\frac{-U_{2 k n} V_{k}-U_{k} V_{2 k n}}{2}=-U_{k(2 n+1)} .
\end{aligned}
$$

Since only the first solution gives positive, considering Binet formulas and recurrence relations of $\left\{U_{k n}\right\}$ and $\left\{V_{k n}\right\}$, we write

$$
n=g^{2}-h^{2}=U_{k(2 n-1)}^{2}-U_{2 k n}^{2}=-\frac{U_{k}^{2}}{D}\left(V_{k} V_{k(4 n-1)}-4\right),
$$

and using $m=1$ in Lemma 2.1,

$$
\begin{aligned}
t & =g^{2}+h^{2}=U_{k(2 n-1)}^{2}+U_{2 k n}^{2}=\frac{U_{k}^{2}}{D}\left(V_{k(4 n-2)}+V_{4 k n}\right) \\
& =U_{k} U_{k(4 n-1)} .
\end{aligned}
$$

Thus, using $x=\left(-V_{k} n-2 U_{k}^{2} \pm t\right) / 2 n$, we obtain the solutions as claimed.

For example, when $k=p=1$ in Theorem 2.5, rational solutions to

$$
n x^{2}+(n+2) x-(n-3)=0
$$

exist if and only if $n=\frac{1}{5}\left(4-L_{4 n-1}\right)$ and they are

$$
\frac{L_{4 n-1}+5 F_{4 n-1}-14}{2\left(4-L_{4 n-1}\right)}, \frac{L_{4 n-1}-5 F_{4 n-1}-14}{2\left(4-L_{4 n-1}\right)} .
$$

We have the following theorem by using Lemma 2.3 and combinatoric identities.

Theorem 2.6. For odd number $k$, rational solutions to

$$
n x^{2}+\left(V_{k} n-2 U_{k}^{2}\right) x-\left(n+\left(V_{k}+2\right) U_{k}^{2}\right)=0
$$

exist if and only if $n=\frac{U_{k}^{2}}{D}\left(V_{k} V_{k(4 n+3)}-4\right)$ and they are

$$
-\frac{V_{k}}{2}+\frac{D}{V_{k} V_{k(4 n+3)}-4} \pm \frac{D V_{k} U_{k(4 n+3)}}{2 U_{k}\left(V_{k} V_{k(4 n+3)}-4\right)} .
$$

For example, when $k=p=1$ in Theorem 2.6, rational solutions to

$$
n x^{2}+(n-2) x-(n+3)=0
$$

exist if and only if $n=\frac{1}{5}\left(L_{4 n-3}-4\right)$ and they are

$$
\frac{10 F_{4 n+3}-L_{4 n+3}+14}{2\left(L_{4 n+3}-4\right)}, \frac{-10 F_{4 n+3}-L_{4 n+3}+14}{2\left(L_{4 n+3}-4\right)} .
$$


Theorem 2.7. For odd number $k$, rational solutions to

$$
n x^{2}+\left(n V_{k}-2 U_{k}^{2} D\right) x-\left(n+D U_{k}^{2}\left(V_{k}+2\right)\right)=0
$$

exist if and only if $n=U_{k}^{2}\left(V_{k} V_{k(4 n+1)}-4\right)$ and they are

$$
x_{1}=\frac{2 V_{k}+D-V_{k} V_{k(4 n+2)}}{\left(V_{k} V_{k(4 n+1)}-4\right)} \text { and } x_{2}=\frac{2 V_{k}+D+V_{k} V_{4 k n}}{\left(V_{k} V_{k(4 n+1)}-4\right)} \text {. }
$$

Proof. The discriminant of (3) is

$$
\Delta_{1}=V_{k}^{2} n^{2}+4\left(n+U_{k}^{2} D\right)^{2} .
$$

Rational solutions of (3) exist if and only if $\Delta_{1}$ is a perfect square, $\Delta_{1}=V_{k}^{2} t^{2}$. Hence,

$$
n^{2}+4\left(\frac{U_{k}^{2} D}{V_{k}}+\frac{n}{V_{k}}\right)^{2}=t^{2}
$$

Thus, $\left[n, \frac{2\left(n+U_{k}^{2} D\right)}{V_{k}}, t\right]$ form Pythagorean triplet. Considering the triplet as

$$
\left[g^{2}-h^{2}, 2 g h, g^{2}+h^{2}\right]
$$

we have

$$
n=g^{2}-h^{2}, \quad \frac{U_{k}^{2}}{V_{k}} D+\frac{n}{V_{k}}=g h, \quad t=g^{2}+h^{2},
$$

and hence

$$
g^{2}-V_{k} h g-h^{2}+U_{k}^{2} D=0 .
$$

From the discriminant of this equation, taking $h=U_{k} h_{1}$, we get

$$
U_{k}^{2} D\left(h_{1}^{2}-4\right)=s^{2}
$$

which has positive solutions $h_{1}=V_{2 k n}$ and $s=D U_{2 k n}$ in Lemma 2.4. Hence

$$
\begin{aligned}
h=U_{k} V_{2 k n}, & \\
g=\frac{h V_{k} \pm s}{2} & =\frac{V_{2 k n} U_{k} V_{k} \pm D U_{2 k n}}{2} \\
& =\frac{V_{k}\left(V_{2 k n} U_{k} \pm V_{k} U_{2 k n}\right)}{2}+2 U_{2 k n} .
\end{aligned}
$$

Taking $m=1$ and $2 n, 2 n-1$ instead of $n$ in Lemma 2.1, respectively, and Binet formula of $\left\{U_{k n}\right\}$,

$$
\begin{aligned}
& g=V_{k} U_{k(2 n+1)}+2 U_{2 k n}=U_{k} V_{k(2 n+1)}, \\
& g=V_{k} U_{k(2 n-1)}-2 U_{2 k n}=-V_{k} U_{k(2 n-1)} .
\end{aligned}
$$

Only the first solution gives positive $n$. Using Lemma 2.1 and Binet formula, recurrence relation of $\left\{V_{k n}\right\}$, we write

$$
n=g^{2}-h^{2}=U_{k}^{2} V_{k(2 n+1)}^{2}-U_{k}^{2} V_{2 k n}^{2}=U_{k}^{2}\left(V_{k} V_{k(4 n+1)}-4\right) .
$$


and by (1)

$$
\begin{aligned}
t & =g^{2}+h^{2}=U_{k}^{2} V_{k(2 n+1)}^{2}+U_{k}^{2} V_{2 k n}^{2} \\
& =U_{k}^{2}\left(V_{k(2 n+1)}^{2}+V_{2 k n}^{2}\right)=U_{k}^{2}\left(V_{k(4 n+2)}+V_{4 k n}\right)=D U_{k} U_{k(4 n+1)}
\end{aligned}
$$

Thus, from $x=\left(-n V_{k}+2 U_{k}^{2} D \pm t\right) / 2 n$, we obtain the solutions as claimed.

For example, when $k=p=1$ in Theorem 2.7, rational solutions to

$$
n x^{2}+(n-10) x-(n+15)=0
$$

exist if and only if $n=L_{(4 n+1)}-4$ and they are

$$
x_{1}=\frac{7-L_{4 n+2}}{L_{4 n+1}-4} \text { and } x_{2}=\frac{7+L_{4 n}}{L_{4 n+1}-4} .
$$

We have the following theorem by using Lemma 2.4 and combinatoric identities.

Theorem 2.8. For odd number $k$, rational solutions to

$$
n x^{2}+\left(V_{k} n+2 U_{k}^{2} D\right) x-\left(n-U_{k}^{2} D\left(V_{k}-2\right)\right)=0
$$

exist if and only if $n=U_{k}^{2}\left(V_{k} V_{k(4 n+1)}-4\right)$ and they are

$$
x_{1}=\frac{2 V_{k}-D-V_{k} V_{k(4 n+2)}}{V_{k} V_{k(4 n+1)}-4} \text { and } x_{2}=\frac{2 V_{k}-D+V_{k} V_{4 k n}}{V_{k} V_{k(4 n+1)}-4} .
$$

For example, rational solutions to

$$
n x^{2}+(n+10) x-(n+5)=0
$$

exist if and only if $n=L_{4 n+1}-4$ and they are

$$
x_{1}=\frac{-3-L_{4 n+2}}{L_{4 n+1}-4} \text { and } x_{2}=\frac{-3+L_{4 n}}{L_{4 n+1}-4} .
$$

\section{References}

[1] Horadam, A. F. (1965). Basic properties of a certain generalized sequence of numbers, The Fib. Quart., 3 (3), 161-176.

[2] Kılıç, E., \& Ömür, N. (2010). Conics characterizing the generalized Fibonacci and Lucas sequences with indices in arithmetic progressions, Ars Com., 94, 459-464.

[3] Kılıç, E., \& Stanica, P. (2009). Factorizations and representations of second linear recurrences with indices in arithmetic progressions, Bol. Mex. Math. Soc., 15 (3), 23-35.

[4] K1lıç, E., \& Stanica, P. (2011). Factorizations of binary polynomial recurrences by matrix methods, Rocky Mount. J. Math., 41 (4), 1247-1264. 
[5] Long, C., Cohen, G., \& Langtry, T. (1993). Arithmetic sequences and second order recurrences, Appl. of Fib. Num., 5, 449-458.

[6] Mahanthappa, M. K. (1991). Arithmetic sequences and Fibonacci quadratics, The Fib. Quart., 29 (4), 343-346.

[7] Umansky, H. (1973). A Fibonacci quadratic, The Fib. Quart., 11, 221-222. 